typeset using JPSJ.sty < ver.1.0b>

\title{
Order $N$ Monte Carlo Algorithm for Fermion Systems Coupled with Fluctuating Adiabatical Fields
}

\author{
Nobuo Furukawa and Yukitoshi Motome ${ }^{1}$ \\ Department of Physics, Aoyama Gakuin University, Fuchinobe 5-10-1, Sagamihara, Kanagawa 229-8558 \\ ${ }^{1}$ RIKEN (The Institute of Physical and Chemical Research), 2-1 Hirosawa, Wako, Saitama 351-0198
}

(Received June 25, 2018)

\begin{abstract}
An improved algorithm is proposed for Monte Carlo methods to study fermion systems interacting with adiabatical fields. To obtain a weight for each Monte Carlo sample with a fixed configuration of adiabatical fields, a series expansion using Chebyshev polynomials is applied. By introducing truncations of matrix operations in a systematic and controlled way, it is shown that the cpu time is reduced from $O\left(N^{3}\right)$ to $O(N)$ where $N$ is the system size. Benchmark results show that the implementation of the algorithm makes it possible to perform systematic investigations of critical phenomena using system-size scalings even for an electronic model in three dimensions, within a realistic cpu timescale.
\end{abstract}

KEYWORDS: Monte Carlo method, Order $N$ method, moment expansion, Chebyshev polynomials, parallel computation, double-exchange model, critical phenomena

\section{$\S 1$. Introduction}

There exists a lot of interests in the class of strongly correlated electron systems where itinerant electrons are coupled to adiabatical fields. An example is the strongly coupled electron-lattice system, while the other is the double-exchange system where electrons are coupled to localized classical spins. Models which represent dilute magnetic semiconductors also belong to this class.

Ground states of such systems may be studied by mean-field approaches, since fluctuations are frozen and irrelevant. However, in order to study finite temperature properties, especially near the critical temperature, it is necessary to take into account fluctuations of the fields. Natures of the field fluctuations are important to understand critical phenomena as well as changes of electronic properties around critical points.

Since dynamics of the fluctuations are adiabatically slow, electrons can respond to the fluctuating potentials precisely so that the electronic states are far from those without fluctuations. Therefore, in the presence of critical fluctuations which are strongly coupled to electrons, various theoretical methods such as mean-field approaches and perturbation theories are invalid.

Numerical studies provide promising methods to calculate such systems. Especially, Monte Carlo (MC) method is suited for calculation of these models. The advantage of this method is that it is possible to obtain thermodynamics of the model on a finite size lattice by taking partition sums for fluctuating fields which are replaced by stochastic samplings. ${ }^{1)}$

However, MC studies suffer from finite-size effects since the system size is limited, due to an increase of the computational complexities and hence cpu time as system sizes are increased. In order to study thermodynamic properties of the model properly, it is requisite to perform extrapolations to the thermodynamic limit as well as finite size scalings. Namely, systematic calculations for various lattice sizes which are large enough for analyses are necessary. In the conventional algorithm, ${ }^{1)}$ the computational complexity scales as $O\left(N^{4}\right)$, where $N$ is the system size. Therefore, it is extremely difficult to increase the system size.

In order to overcome the difficulty, improved algorithms have been proposed to reduce the computational complexity for the calculation of the Boltzmann weight. The authors have introduced the polynomial expansion method (PEM), where the computational complexity is reduced to $O\left(N^{3}\right) .^{2,3)}$ Alonso et al. ${ }^{4}$ have applied the hybrid $\mathrm{MC}$ algorithm which makes the computational complexity to scale as $O\left(N^{2}\right)$. Using these new methods, larger system sizes become available. As an example, critical phenomena of the two-dimensional doubleexchange model have been investigated using finite-size scaling analysis and non-equilibrium relaxation studies. ${ }^{5,6)}$ However, these algorithms are still not sufficient enough to study systems which require much larger computational scales, such as models with more complex interactions or those in three dimensions.

In this paper, we present a new algorithm which further reduces the computational complexity of the $\mathrm{MC}$ calculations to $O(N)$. In $\S 2$, we briefly describe the PEM in order to make this article self-contained. In $\S 3$, we introduce a truncation method to improve the PEM. Benchmark results and estimates of the truncation errors are shown in $\S 4$. Sec. 5 is devoted to summary and discussions.

\section{§2. Polynomial Expansion Monte Carlo Method}

\subsection{Hamiltonian matrices}

Throughout this paper, we consider a system where the Hamiltonian operator is expressed in a quadratic 
form,

$$
\hat{\mathcal{H}}(\phi)=\sum_{i j} c_{i}^{\dagger} H_{i j}(\phi) c_{j} .
$$

Here, $c_{i}\left(c_{i}^{\dagger}\right)$ represents a fermion annihilation (creation) operator for an index $i$. Each index represents fermionic degrees of freedom, typically a combination of site, orbital and spin. Matrix elements depend on the adiabatical fields which are expressed by $\phi$. This means that we restrict ourselves to a class of electronic systems on lattices coupled to adiabatical fields which give, e.g., charge/spin density potentials as well as those coupled to orbital degrees of freedom as in Jahn-Teller couplings. We assume the absence of electron-electron interactions.

Later in this paper, simple examples of the Hamiltonian (1) will be given in Eq. (22), where spinless electrons are coupled to on-site potential fields, and in Eq. (41) where electrons are coupled to localized spins defined on each site in such a way that transfer energies are modulated by configurations of the spins.

Let us describe the class of systems which are expressed by the Hamiltonian (1) more precisely. In usual cases, diagonal elements of the Hamiltonian matrices describe potential energies, e.g., charge density potentials coupled to adiabatical fields. Electron hopping terms, as well as coupling to transverse fields give off-diagonal matrix elements of $H$. Within the scheme we do not consider types of fields which break electron number conservation, e.g., coupling to singlet superconducting fields in a form $\Delta_{i} c_{i \uparrow}^{\dagger} c_{i \downarrow}^{\dagger}+h . c$., unless there exists a canonical transformation which maps the system to a particlenumber conserving system, e.g., $c_{i \uparrow} \rightarrow d_{i \uparrow}$ and $c_{i \downarrow} \rightarrow d_{i \downarrow}^{\dagger}$ in the previous example.

For a fixed configuration of the adiabatical fields, the Hamiltonian in Eq. (1) shows a one-body electron system with random potentials. The Hamiltonian matrices $H$ have a matrix dimension $N_{\text {dim }}$ defined by the total number of fermionic degrees of freedom, which is proportional to the system size $N$. Within this article we restrict ourselves to the cases where $H$ are sparse matrices, namely, the model has short range hoppings in usual cases.

We assume that the adiabatical fields are locally defined, typically on sites or bonds. Then, the total number of the adiabatical fields is proportional to $N$. We also restrict ourselves to the case where interactions between the fields and the electrons are short-ranged, i.e., the number of matrix elements which are modulated by the change of an adiabatical field is $O\left(N^{0}\right)$.

\subsection{Boltzmann weight}

The partition function for the Hamiltonian (1) is written as

$$
Z=\operatorname{Tr}_{\mathrm{C}} \operatorname{Tr}_{\mathrm{F}} \exp \left(-\beta\left[\hat{\mathcal{H}}(\phi)-\mu \hat{N}_{\mathrm{e}}\right]\right),
$$

where $\operatorname{Tr}_{\mathrm{C}}$ is the trace over adiabatical fields $\phi$, while $\operatorname{Tr}_{\mathrm{F}}$ is the grand canonical trace over fermion degrees of freedom. Here, $\beta$ is the inverse temperature, $\mu$ is the chemical potential and $\hat{N}_{\mathrm{e}}$ is the particle-number operator.
In MC approaches, the trace over adiabatical fields is replaced by the stochastical sampling of the field configurations $\phi$ whose Boltzmann weight is given by

$$
P(\phi)=\frac{1}{Z} \exp \left[-S_{\text {eff }}(\phi)\right] .
$$

Here, the effective action $S_{\text {eff }}$ is defined by

$$
\begin{aligned}
S_{\text {eff }}(\phi) & \equiv-\log \left(\operatorname{Tr}_{\mathrm{F}} \mathrm{e}^{-\beta\left[\hat{\mathcal{H}}(\phi)-\mu \hat{N}_{\mathrm{e}}\right]}\right) \\
& =\sum_{\nu=1}^{N_{\mathrm{dim}}} F\left(\varepsilon_{\nu}(\phi)\right)
\end{aligned}
$$

where

$$
F(x)=-\log \left[1+\mathrm{e}^{-\beta(x-\mu)}\right],
$$

while $\varepsilon_{\nu}$ is the $\nu$-th eigenvalue of the Hamiltonian matrix $H$ for a given configuration of $\phi$.

In an importance sampling MC method, probability of an update from an old field configuration $\phi^{\text {old }}$ to a new configuration $\phi^{\text {new }}$ depends on the ratio of the Boltzmann weights which is given by

$$
r=\frac{P\left(\phi^{\text {new }}\right)}{P\left(\phi^{\text {old }}\right)} .
$$

In a local update of the adiabatical fields, we generate $\phi^{\text {new }}$ from $\phi^{\text {old }}$ in such a way that only one or a local group of adiabatical fields is modified from $\phi^{\text {old }}$ but the rest are unchanged. The definition of a MC step with local updates is that we make a sweep of local updates so that all adiabatical field variables are sequentially examined for updates.

One of the method to calculate the Boltzmann weight $P$ from Eqs. (3)-(5) is the diagonalization method (DM) where $\varepsilon_{\nu}(\phi)$ are exactly obtained by direct diagonalizations of the Hamiltonian matrix $H(\phi) .{ }^{1}{ }^{1}$ The computational complexity for each matrix diagonalization to obtain all eigenvalues is $O\left(N_{\mathrm{dim}}{ }^{3}\right)$. In a MC step with local updates, the number of trials for the field upgrades scales as $O(N)$. Since $N_{\text {dim }} \propto N$, the total computational complexity for a MC step by the DM is $O\left(N^{4}\right)$.

\subsection{Polynomial expansion method}

An approach to reduce the computational complexity in the calculation of $P(\phi)$ is to perform a polynomial expansion. ${ }^{2)}$ When $F(x)$ in Eq. (5) is expanded by a series of polynomials $\left\{T_{m}(x)\right\}$ in a form

$$
F(x)=\sum_{m=0}^{\infty} f_{m} T_{m}(x),
$$

we may rewrite Eq. (4) as

$$
S_{\mathrm{eff}}(\phi)=\sum_{m} f_{m} \mu_{m},
$$

where $\mu_{m}$ is the polynomial moments of the Hamiltonian defined by

$$
\mu_{m}=\sum_{\nu=1}^{N_{\mathrm{dim}}} T_{m}\left(\varepsilon_{\nu}(\phi)\right)=\operatorname{Tr} T_{m}(H(\phi)) .
$$


Here Tr represents a trace operation for the matrix polynomials. The values of $f_{m}$ depend on temperature and chemical potential but not on the adiabatical fields $\phi$. Moments $\mu_{m}$ depend on $\phi$, which are calculated through trace operations and matrix addition/multiplications of $H(\phi)$. Therefore, the expansion of the effective action $S_{\text {eff }}$ by a polynomial series enables us to obtain Boltzmann weights for each update of the adiabatical fields through simple matrix operations only.

Among various choices of polynomials for the series expansion in Eq. (7), the Chebyshev polynomials ${ }^{7,8)}$ give us an advantage that the expansion coefficients $f_{m}$ decay quickly for $m \gg 1$ in an exponential way. ${ }^{2}$ ) The Chebyshev polynomials $\left\{T_{m}(x)\right\}$ for $m=0,1, \ldots$ are recursively defined by

$$
\begin{aligned}
T_{0}(x) & =1, \quad T_{1}(x)=x, \\
T_{m}(x) & =2 x T_{m-1}(x)-T_{m-2}(x) .
\end{aligned}
$$

Within the region $-1 \leq x \leq 1$, the Chebyshev polynomials show an orthonormal property in a form

$$
\int_{-1}^{1} \frac{\mathrm{d} x}{\pi \sqrt{1-x^{2}}} T_{m}(x) T_{m^{\prime}}(x)=\alpha_{m} \delta_{m m^{\prime}},
$$

where

$$
\alpha_{m}= \begin{cases}1, & m=0, \\ 1 / 2, & m \neq 0 .\end{cases}
$$

Using this relation, the coefficients in Eq. (7) are obtained as

$$
f_{m}=\frac{1}{\alpha_{m}} \int_{-1}^{1} \frac{\mathrm{d} x}{\pi \sqrt{1-x^{2}}} F(x) T_{m}(x) .
$$

In Fig. 1 we show some examples for absolute values of the coefficients $\left|f_{m}\right|$ where we see exponential decays.

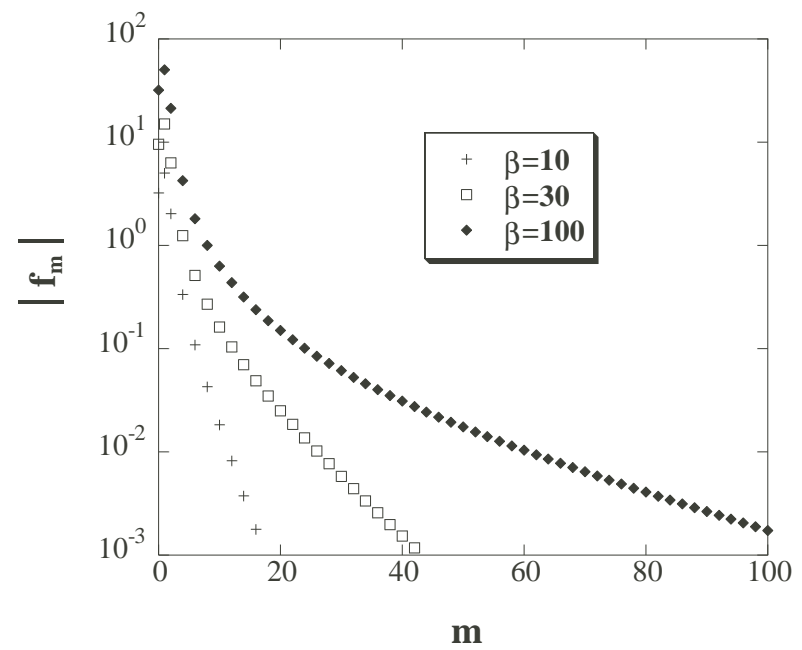

Fig. 1. Absolute values of the coefficient $\left|f_{m}\right|$ for the Chebyshev polynomial expansion of $S_{\text {eff }}$, for $\beta=10,30$ and 100 at $\mu=0$.

From Eq. (9) we have

$$
\mu_{m}=\int_{-1}^{1} \mathrm{~d} \varepsilon D_{\phi}(\varepsilon) T_{m}(\varepsilon)
$$

$$
=\int_{0}^{\pi} \mathrm{d} \theta \sin \theta D_{\phi}(\theta) \cos m \theta
$$

where

$$
D_{\phi}(\varepsilon)=\sum_{\nu} \delta\left(\varepsilon-\varepsilon_{\nu}(\phi)\right)
$$

is the density of states (DOS) for the eigenvalues of $H(\phi)$. Here we used an alternative definition of the Chebyshev polynomials,

$$
T_{m}(\cos \theta)=\cos m \theta \text {. }
$$

We note here that, since Chebyshev polynomials $T_{m}(x)$ as an orthonormal set are defined in the region $-1 \leq$ $x \leq 1$, the Hamiltonian matrices have to be scaled properly so that the eigenvalues satisfy $-1 \leq \varepsilon_{\nu}(\phi) \leq 1$ for $\nu=1, \ldots, N_{\text {dim }}$. From Eq. (14) we see that $\mu_{m}$ is a Fourier transform of $\sin \theta D_{\phi}(\cos \theta)$. This means that $\left|\mu_{m}\right|$ either converge to zero at $m \gg 1$ if the DOS is non-singular, or converge to a constant in the most extreme cases where there exist macroscopic degeneracies in the DOS. Therefore, the series $f_{m} \mu_{m}$ in Eq. (8) decays exponentially.

Replacement of the infinite sum over $m$ in Eq. (8) by a finite sum up to $m=M$ gives us accurate results if an appropriate value of $M$ is chosen. Although such a value depends on an asymptotic form of $\mu_{m}$ which is not predictable a priori, we can always estimate truncation errors of PEM due to finite $M$ through comparisons between the results by the PEM and the DM. Note that, even when it is difficult to perform a product run by the DM with large enough MC steps to obtain statistically accurate results, it is usually possible to execute a few trial MC steps in order to estimate truncation errors. In $\mathrm{MC}$ runs in practice, truncation errors can be neglected if they are sufficiently smaller than the statistical errors.

\subsection{Algorithm}

The algorithm for the PEM using the Chebyshev polynomials is as follows. We introduce a set of orthonormal vectors $\{\vec{e}(k)\}\left(k=1, \ldots, N_{\operatorname{dim}}\right)$, and calculate

$$
\vec{v}(k, m) \equiv T_{m}(H) \vec{e}(k),
$$

for $m \geq 0$. Then, the trace $\mu_{m}$ is given by

$$
\mu_{m}=\sum_{k=1}^{N_{\text {dim }}} \mu_{m}(k)
$$

where

$$
\mu_{m}(k)=(\vec{e}(k), \vec{v}(k, m)) .
$$

Here ( , ) represents an inner product.

Using a recursion relation for the Chebyshev polynomials in Eq. (10), we obtain $\vec{v}(k, m)$ as

$$
\vec{v}(k, 0)=\vec{e}(k), \quad \vec{v}(k, 1)=H \vec{v}(k, 0),
$$

and

$$
v_{i}(k, m)=2 \sum_{j} H_{i j} v_{j}(k, m-1)-v_{i}(k, m-2),
$$

for $i=1, \ldots, N_{\operatorname{dim}}$ at $m \geq 2$, where $v_{i}(k, m)$ is the $i$-th 
element of the vector $\vec{v}(k, m)$.

The summation $\sum_{j}$ in Eq. (21) can be restricted to $j$ with non-zero matrix elements $H_{i j} \neq 0$. Since we assume sparse matrices in Eq. (21), computational complexity of these matrix operations are $O(N)$. Therefore, for a fixed configuration of $\phi$, the computational complexity to obtain $\mu_{m}(k)(0 \leq m \leq M)$ is $O(M N)$, while that for the trace operation is $O(N)$. Then, the complexity of the Boltzmann weight calculation scales as $O\left(M N^{2}\right)$. This means that, for one MC step with local updates where $O(N)$ field variables are manipulated sequentially, the computational complexity is $O\left(M N^{3}\right)$. Compared to the DM which scales as $O\left(N^{4}\right)$, the PEM is advantageous if $M \ll N$.

It has also been shown that the algorithm is suited for parallel computations since trace operations are mutually independent, ${ }^{2,9)}$ which further reduce elapsed time for calculations. As a result, it become possible to investigate models with larger system sizes within a reasonable scale of cpu time. Using the PEM, critical phenomena at finite temperatures for a fermionic model in two dimensions are studied for the first time by finite-size scaling analysis as well as by non-equilibrium relaxation technique. $^{5,6)}$ However, the algorithm still turns out to be insufficient to study critical phenomena in three dimensions, since the reduction of the computational complexities is not large enough.

\section{§3. Truncated Polynomial Expansion Method}

Now we demonstrate that the calculations for the Boltzmann weights can further be improved by introducing truncated matrix operations. As an example to explain the method, let us first consider a simple model which has the Hamiltonian matrix in the form

$$
H_{i j}(\phi)= \begin{cases}g \phi_{i} & i=j \\ -t & i \text { and } j \text { are nearest neighbors } \\ 0 & \text { otherwise. }\end{cases}
$$

Here $t$ is the nearest neighbor hopping energy for spinless electrons while $g$ is the electron-field coupling constant. In this system, local adiabatical field $\phi=\left\{\phi_{i}\right\}$ is defined on each lattice which is coupled to electrons as an onsite potential. The Hamiltonian matrix $H(\phi)$ is a sparse matrix with $N_{\text {dim }}=N$.

\subsection{Truncation of matrix products}

In order to obtain $\mu_{m}(k)$ for $m=0, \ldots, M$ from Eqs. (19)-(21), matrix-vector multiplications throughout the Hilbert space are necessary, which give $O(M N)$ computational complexity. Here we introduce a truncation of the matrix-vector multiplications in order to reduce the computational complexity.

Let us choose $e_{i}(k)=\delta_{i k}$ for the orthonormal set in Eq. (17). Since $v_{i}(k, 0)$ is non-zero only at $i=k$, we have $v_{i}(k, 1) \neq 0$ only at $i=k$ as well as at nearest neighbors of $k$. Namely, due to the sparse nature of the Hamiltonian matrix (22), it is not necessary to calculate all the other vector elements. Similarly, if one keeps track of a set of indices with $v_{i}(k, m-1) \neq 0$, the calculation of vector elements $v_{i}(k, m)$ can be restricted to limited numbers of indices, so that the computational complexity is much reduced.

The matrix-vector product in Eq. (21) can be viewed as a transfer-matrix multiplication to a state vector, which expresses a diffusive propagation of a wavefunction. We start from an initial vector $\vec{v}(k, 0)=\vec{e}(k)$, which expresses an electron state localized at site $k$. Each time the Hamiltonian matrix $H$ is multiplied to obtain $\vec{v}(k, m)$, electrons hop to nearest neighbors. As a consequence, the sites with non-zero vector elements $v_{i}(k, m)$ spread out as $m$ increases. In Fig. 2 we give a schematic illustration for the propagation steps. The process also resembles the diffusion of the probability distribution function in a random-walk system.

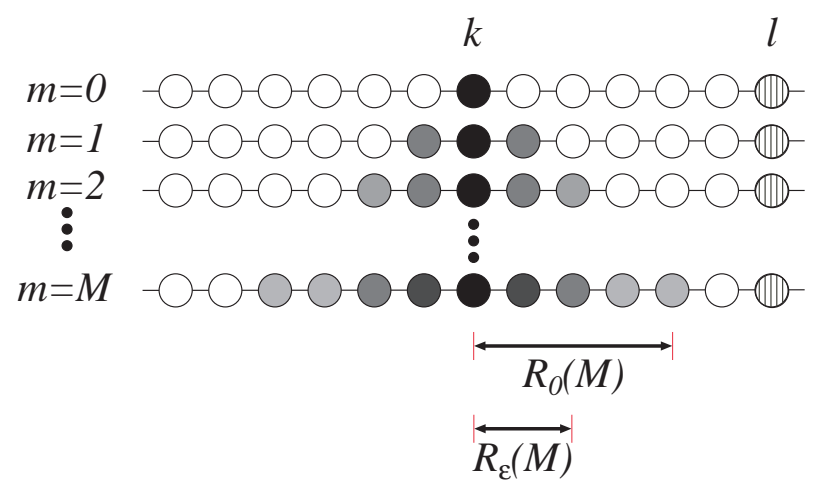

Fig. 2. A sketch for the propagation of vector elements. The vertical axis gives the matrix-vector multiplication steps. Circles aligned in the horizontal direction represent lattice sites. Filled circles show sites with non-zero vector elements, and darkness of them schematically illustrates amplitudes of the vector elements. The initial vector gives a localized state at site $k$, while the hatched circle at $l$ symbolizes the site where the Hamiltonian matrix element is updated. See also the discussion in $\S 3.2$

Let us define the distance between $i$-th site and $k$-th site, denoted as $\|i-k\|$, by the minimum number of hops for an electron to transfer from $i$-th site to $k$-th site. On hypercubic lattices, this gives the "Manhattan distance". We define the range of propagation $R_{0}(m)$ by the longest distance that an electron can hop by $m$ steps of the matrix-vector multiplication in Eq. (21). Since sites which are outside of the range of propagation have zero vector elements, i.e.,

$$
v_{i}(k, m)=0 \quad \text { if }\|i-k\|>R_{0}(m),
$$

we have no contribution to the calculations of $\mu_{m}(k)$ as well as $\vec{v}(k, m+1)$ from these sites. This means that we may perform our matrix-vector calculation only within the neighbors of $k$ which satisfies $\|i-k\| \leq R_{0}(m)$ to obtain exact results. In the present model (22) we have $R_{0}(m)=m$, and the number of sites which contributes to the overall calculation of $\mu_{m}(k)(m \leq M)$ is proportional to $M^{d}$ instead of $N$ when every sites on the lattice is taken into account. Here $d$ is the spacial dimension of the lattice. By introducing this restriction, the computational complexity to obtain $\mu_{m}(k)(m \leq M)$ is reduced from $O(M N)$ to $O\left(M^{d+1}\right)$ without any cost for the computational accuracies. 
We can further reduce the range where calculations are restricted, by introducing a threshold $\epsilon$ for the vector elements. When the absolute values of the vector elements $\left|v_{i}(k, m)\right|$ are small enough, we can neglect such terms in the calculation of $\mu_{m}(k)$ and $\vec{v}(k, m+1)$. Let us define the range of propagation $R_{\epsilon}(m)$ by the longest distance $\|i-k\|$ such that the absolute value of the vector element on the $i$-th site exceeds the threshold, $\left|v_{i}(k, m)\right| \geq \epsilon$. In Fig. 2 we give a schematic illustration. Then, we have

$$
\left|v_{i}(k, m)\right|<\epsilon \quad \text { if }\|i-k\|>R_{\epsilon}(m),
$$

and contributions to $\mu_{m}(k)$ from sites outside of the range are negligible if we take values of $\epsilon$ appropriately.

Since the diffusion length is proportional to the squareroot of the time-steps in general, $R_{\epsilon}(m) \propto \sqrt{m}$ for $\epsilon>0$. Then, by introducing a threshold $\epsilon$ and restricting the calculation within $\|i-k\| \leq R_{\epsilon}(m)$, the number of sites which contributes to $\mu_{m}(k)$ scales as $O\left(M^{d / 2}\right)$. Therefore the computational complexity to obtain $\mu_{k}(m)(m \leq M)$ is further reduced to $O\left(M^{d / 2+1}\right)$ with the accuracies of $O(\epsilon)$. (To be more specific, the number of sites neglected by this treatment is $O\left(M^{d}\right)$ for the calculation of $\mu_{m}$ $(m=0, \ldots, M)$, so the total error for the calculation of the Boltzmann weight is $O\left(M^{d+1} \epsilon\right)$.)

Let us now extend the procedure to general cases where an index for fermion degrees of freedom represents a combination of site, orbital and spin. Since the basis set to define the Hamiltonian matrices (1) can be taken arbitrary, we may also consider a system where it is not well-defined to consider a geometrical distance between indices. Nevertheless, as long as the Hamiltonian matrices are sparse, we can generalize the algorithm as follows.

We define a subspace $\mathcal{N}_{0}(k, m)$ as a set of neighboring indices of the initial index $k$ which are within the range of propagation by $m$ steps,

$$
\mathcal{N}_{0}(k, m)=\bigcup_{m^{\prime}=0}^{m}\left\{i \mid v_{i}\left(k, m^{\prime}\right) \neq 0\right\} .
$$

Note that, in the previous example, $\mathcal{N}_{0}(k, m)$ is a set of indices within the range of $R_{0}(m)$ from $k$. Then, we may restrict the matrix product operations within the subspace $\mathcal{N}_{0}(k, m)$, since

$$
v_{i}(k, m)=0 \quad \text { if } i \notin \mathcal{N}_{0}(k, m),
$$

so that there is no contribution to $\mu_{m}(k)$ from outside of $\mathcal{N}_{0}(k, m)$.

Similarly, we define a subspace $\mathcal{N}_{\epsilon}(k, m)$ as a set of neighboring indices of $k$ where absolute values of the vector elements exceed the threshold $\epsilon$,

$$
\mathcal{N}_{\epsilon}(k, m)=\bigcup_{m^{\prime}=0}^{m}\left\{i|| v_{i}\left(k, m^{\prime}\right) \mid>\epsilon\right\} .
$$

In the previous example, $\mathcal{N}_{\epsilon}(k, m)$ roughly corresponds to a set of sites within the range of $R_{\epsilon}(m)$ from $k$. By making truncations of the matrix product operations within the restricted subspace $\mathcal{N}_{\epsilon}(k, m)$, we obtain approximate results for $\mu_{m}(k)$ within errors of $O(\epsilon)$, since

$$
\left|v_{i}(k, m)\right|<\epsilon \quad \text { if } i \notin \mathcal{N}_{\epsilon}(k, m),
$$

so that contributions from outside of $\mathcal{N}_{\epsilon}(k, m)$ are negligible.

\subsection{Truncation of trace operations}

In order to obtain $S_{\text {eff }}$ directly from Eqs. (8) and (9), a trace operation throughout the Hilbert space is necessary, which gives $O(N)$ computational complexity. Here we introduce a truncation of the trace operation in order to reduce the computational complexity.

The probability of the MC update from an old field configuration $\phi^{\text {old }}$ to a new configuration $\phi^{\text {new }}$, which is given by the ratio of the Boltzmann weights in Eq. (6), can be calculated from

$$
\frac{P\left(\phi^{\text {new }}\right)}{P\left(\phi^{\text {old }}\right)}=\exp \left(-\Delta S_{\text {eff }}\right),
$$

where $\Delta S_{\text {eff }}$ is the difference of the effective action. Using PEM up to the $M$-th order, we have

$$
\begin{aligned}
\Delta S_{\mathrm{eff}} & =S_{\mathrm{eff}}\left(\phi^{\text {new }}\right)-S_{\mathrm{eff}}\left(\phi^{\text {old }}\right) \\
& \simeq \sum_{m=0}^{M} f_{m} \sum_{k}^{N_{\mathrm{dim}}} \Delta \mu_{m}(k),
\end{aligned}
$$

where

$$
\begin{aligned}
\Delta \mu_{m}(k)=\left(\vec{e}(k), \vec{v}^{\text {new }}(k, m)\right) \\
-\left(\vec{e}(k), \vec{v}^{\text {old }}(k, m)\right) .
\end{aligned}
$$

Here, $\vec{v}^{\alpha}(k, m)$ for $\alpha=$ (old, new) is defined by

$$
\vec{v}^{\alpha}(k, m)=T_{m}\left(H\left(\phi^{\alpha}\right)\right) \vec{e}(k),
$$

and we choose $e_{i}(k)=\delta_{i k}$. Summation over $k$ in Eq. (30) is the trace operation in Eq. (9).

Now we consider a local update of the adiabatical fields in the present exemplified model (22). Let us choose a site $l$ and try a local update on the site $\phi_{l}^{\text {old }} \rightarrow \phi_{l}^{\text {new }}$, while we have $\phi_{i}^{\text {old }}=\phi_{i}^{\text {new }}$ for $i \neq l$. In this case, the change of the Hamiltonian matrix $H\left(\phi^{\text {old }}\right) \rightarrow H\left(\phi^{\text {new }}\right)$ exists only at the $l, l$-th matrix element, while we have $H_{i j}\left(\phi^{\text {old }}\right)=H_{i j}\left(\phi^{\text {new }}\right)$ elsewhere.

Let us take a site $k$ which is distant enough from the updated site $l$ so that $\|k-l\|>R_{0}(M)$ is satisfied, and consider the diffusion of the vectors $\vec{v}^{\text {old }}(k, m)$ and $\vec{v}^{\text {new }}(k, m)$. In this case, we have $\vec{v}^{\text {old }}(k, m)=\vec{v}^{\text {new }}(k, m)$ and hence $\Delta \mu_{m}(k)=0$. The reason is as follows. For $m \leq M$, the region where the state vectors propagate does not reach the site $l$, since $R_{0}(m)<\|k-l\|$ is satisfied. In Fig. 2 we give a schematic illustration. The matrix elements that are operated to the vectors during the diffusion processes are identical between old and new configurations. This makes

$$
v_{i}^{\text {old }}(k, m)=v_{i}^{\text {new }}(k, m)
$$

for $i$ which satisfies $\|i-k\| \leq R_{0}(m)$. At the same time, by the definition of $R_{0}(m)$ we have $v_{i}^{\text {old }}(k, m)=0$ and $v_{i}^{\text {new }}(k, m)=0$ for $i$ such that $\|i-k\|>R_{0}(m)$. Then, we have $v_{i}^{\text {old }}(k, m)=v_{i}^{\text {new }}(k, m)$ in the entire space.

In other words, we have $\Delta \mu_{m}(k) \neq 0$ for $m \leq M$ only if $k$ is close enough to $l$ so that the propagation from the site $k$ reaches the site $l$ within $M$ steps. Therefore, it is sufficient to take the summation over $k$ in Eq.(30) only 
within the vicinity of $l$ which satisfies $\|k-l\| \leq R_{0}(M)$. Namely, the trace operation may be restricted to a subspace which has $O\left(M^{d}\right)$ sites so that the computational complexity for the trace operations is reduced from $O(N)$ to $O\left(M^{d}\right)$.

Furthermore, we introduce a truncation of trace operations which gives approximate results with a reduced computational complexity. Here we consider a general case with sparse Hamiltonian matrices. For a moment we restrict ourselves to an update of the adiabatical field where the matrix elements of $H\left(\phi^{\text {old }}\right)$ and $H\left(\phi^{\text {new }}\right)$ are identical except for the $l, l$-th element.

Let us consider an initial vector at $k$ and the propagation of the vectors for $H\left(\phi^{\text {old }}\right)$ and $H\left(\phi^{\text {new }}\right)$, which gives the set of neighboring indices $\mathcal{N}_{\epsilon}^{\text {old }}(k, m)$ and $\mathcal{N}_{\epsilon}^{\text {new }}(k, m)$, respectively. If $l \notin \mathcal{N}_{\epsilon}^{\text {new }}(k, M)$ and $l \notin$ $\mathcal{N}_{\epsilon}^{\text {old }}(k, M)$ are satisfied, both $\vec{v}^{\text {old }}(k, m)$ and $\vec{v}^{\text {new }}(k, m)$ are approximately confined within the subspace where matrix elements of the Hamiltonian are identical, and do not reach the index $l$. Then we have

$$
\vec{v}^{\text {old }}(k, m) \simeq \vec{v}^{\text {new }}(k, m),
$$

within the error threshold $\epsilon$, and therefore $\Delta \mu_{m}(k)=$ $O(\epsilon)$ is satisfied. In other words, only indices in the vicinity of $l$ where effective propagations to $l$ occur within $M$ steps should be considered for the calculations of $\Delta \mu_{m}(k)$.

This means that the trace operation in Eq. (30) can be restricted to the vicinity of $l$, defined by a set of indices $k$ where $l \in \mathcal{N}_{\epsilon}^{\text {new }}(k, M)$ or $l \in \mathcal{N}_{\epsilon}^{\text {old }}(k, M)$ are satisfied. Using Eq. (32) and the Hermicity of the Hamiltonian matrix polynomials

$$
\left.T_{m}(H)\right|_{l k}=\left(\left.T_{m}(H)\right|_{k l}\right)^{*},
$$

we have $v_{l}^{\alpha}(k, m)=v_{k}^{\alpha}(l, m)^{*}$ for $\alpha=$ (old, new). Namely, if and only if $k \in N_{\epsilon}^{\alpha}(l, M)$, we have $l \in$ $N_{\epsilon}^{\alpha}(k, M)$. Therefore, the trace operation can be restricted to a subspace defined by

$$
\mathcal{V}_{\epsilon}(l, M) \equiv \mathcal{N}_{\epsilon}^{\text {old }}(l, M) \cup \mathcal{N}_{\epsilon}^{\text {new }}(l, M) .
$$

Due to the diffusive nature of the propagation, the number of indices in $\mathcal{N}_{\epsilon}^{\text {old }}(l, M)$ and $\mathcal{N}_{\epsilon}^{\text {new }}(l, M)$ is $O\left(M^{d / 2}\right)$ on usual lattice systems. The truncated trace operation within $\mathcal{V}_{\epsilon}$ reduces the computational complexity from $O(N)$ to $O\left(M^{d / 2}\right)$ with errors of $O(\epsilon)$.

We can extend our algorithm to cases where a local update modulates matrix elements for multiple indices. An example is the case where fields are coupled to offdiagonal matrix elements, e.g., hopping amplitudes. Let us consider a case where a local update modulates the $l, l^{\prime}$-th matrix element. Differences between $\vec{v}^{\text {old }}$ and $\vec{v}^{\text {new }}$ exist if the propagations of the vectors reach either of the indices $l$ or $l^{\prime}$. Then, we need to consider a sum of vicinities centered at $l$ and $l^{\prime}$,

$$
\mathcal{V}_{\epsilon}^{\text {tot }}=\mathcal{V}_{\epsilon}(l, M) \cup \mathcal{V}_{\epsilon}\left(l^{\prime}, M\right),
$$

and make trace operations within $\mathcal{V}_{\epsilon}^{\text {tot }}$. In a general case where a number of matrix elements are modulated, we need to consider all the indices associated with modulated matrix elements. We define $\mathcal{C}$ as a set of indices where matrix elements are modulated by the update,

$$
\mathcal{C}=\left\{l \mid{ }^{\exists} l^{\prime}, H_{l l^{\prime}}\left(\phi^{\text {old }}\right) \neq H_{l l^{\prime}}\left(\phi^{\text {new }}\right)\right\} .
$$

Then, the total vicinity $\mathcal{V}_{\epsilon}^{\text {tot }}$ is given by

$$
\mathcal{V}_{\epsilon}^{\text {tot }}=\bigcup_{l \in \mathcal{C}} \mathcal{V}_{\epsilon}(l, M)
$$

and the trace operations are performed within $\mathcal{V}_{\epsilon}^{\text {tot }}$, i.e.,

$$
\Delta S_{\text {eff }} \simeq \sum_{m=0}^{M} f_{m} \sum_{k \in \mathcal{V}_{\epsilon}^{\text {tot }}} \Delta \mu_{m}(k) .
$$

As long as MC updates are local, the number of indices in $\mathcal{C}$ is $O\left(N^{0}\right)$, so that the computational complexity for the trace operation will be $O\left(M^{d / 2}\right)$.

\subsection{Comparison with previous methods}

Thus we see that the PEM using truncated matrix operations reduces the total computational complexity for one local update from $O\left(M N^{2}\right)$ to $O\left(M^{d+1}\right)$, by combining threshold truncations for both matrix products and trace operations. Hereafter we refer to the improved method in this section with truncations of matrix operations as the truncated PEM, whereas the original method described in $\S 2$ is called as the full PEM. In Table I we summarize the computational complexities for various algorithms for comparison.

Table I. Computational complexities to perform calculations of $\mu_{m}(k)$, trace operations, calculations of the Boltzmann weight ratio through $\Delta S_{\text {eff }}$, and a MC step with local updates in total. Here, f-PEM and t-PEM stand for the full PEM and the truncated PEM, respectively. Threshold for the truncated PEM is described by $\epsilon$.

\begin{tabular}{ccccc}
\hline Algorithm & $\mu_{m}(k)$ & Trace & $\Delta S_{\text {eff }}$ & Total \\
\hline DM & - & - & $O\left(N^{3}\right)$ & $O\left(N^{4}\right)$ \\
f-PEM & $O(M N)$ & $O(N)$ & $O\left(M N^{2}\right)$ & $O\left(M N^{3}\right)$ \\
t-PEM & & & & \\
$\epsilon=0$ & $O\left(M^{d+1}\right)$ & $O\left(M^{d}\right)$ & $O\left(M^{2 d+1}\right)$ & $O\left(M^{2 d+1} N\right)$ \\
$\epsilon \neq 0$ & $O\left(M^{\frac{d}{2}+1}\right)$ & $O\left(M^{\frac{d}{2}}\right)$ & $O\left(M^{d+1}\right)$ & $O\left(M^{d+1} N\right)$ \\
\hline
\end{tabular}

Let us emphasize here that the restriction of matrix operations within $\mathcal{N}_{0}(k, M)$ gives us identical results for $\mu_{m}(k)$ to those obtained by the full PEM, with a reduced computational complexity. We also have $\mathcal{N}_{\epsilon \rightarrow 0}(k, M)=\mathcal{N}_{0}(k, M)$. This implies that the introduction of the threshold $\epsilon$ is a controlled approximation in the sense that $\mu_{m}(k)$ are obtained with an arbitrary accuracy by an appropriate choice of $\epsilon$, with further reduced computational complexities.

\section{§4. Algorithm and Implementation}

\subsection{Algorithm}

Now we clarify actual algorithms to perform the truncated PEM. We first show an algorithm for the truncated matrix product to obtain $v_{i}(k, m)$ for $m \leq M$ :

i) Determine the truncation threshold for matrix products $\epsilon_{\mathrm{p}}$. Set the initial unit vector $v_{i}(k, 0)=\delta_{i k}$, and the initial restricted subspace $\mathcal{N}_{\epsilon_{\mathrm{p}}}=\{k\}$. 
ii) From given $\left\{v_{i}(k, m-1)\right\}$ and $\mathcal{N}_{\epsilon_{\mathrm{p}}}$, perform matrixvector product to calculate $\left\{v_{i}(k, m)\right\}$ within restricted subspace. Namely, indices $j$ in Eq. (21) are restricted to $j \in \mathcal{N}_{\epsilon_{\mathrm{p}}}$, whereas indices $i$ also include those generated by propagations due to non-zero offdiagonal matrix elements of $H_{i j}$ in Eq. (21).

iii) The treatment for a newly generated index $i$ is as follows: If $\left|v_{i}(k, m)\right| \geq \epsilon_{\mathrm{p}}$, register $i$ as a new member of $\mathcal{N}_{\epsilon_{\mathrm{p}}}$. Otherwise, discard the calculation for $v_{i}(k, m)$ and treat it as zero.

iv) Repeat steps ii) and iii) $M$ times.

v) As a byproduct, we obtain $\mathcal{N}_{\epsilon_{\mathrm{p}}}(k, M)$.

This procedure automatically gives calculations without truncation errors by setting $\epsilon_{\mathrm{p}}=0$.

An algorithm to perform truncated trace operation for an update of the field is as follows:

i) Determine the truncation threshold for trace operations $\epsilon_{\mathrm{tr}}$.

ii) Define $\mathcal{C}$ as in Eq. (38). Perform truncated matrix product operations to obtain $\vec{v}^{\alpha}(l, M)$ for $l \in \mathcal{C}$ and $\alpha=$ (new, old), so that the neighbors of the localupdated indices $\mathcal{N}_{\epsilon_{\mathrm{tr}}}^{\alpha}(l, M)$ are determined. Then, $\mathcal{V}_{\epsilon_{\text {tr }}}^{\text {tot }}$ is obtained from Eqs. (36) and (39).

iii) For $k \in \mathcal{V}_{\epsilon_{\text {tr }}}^{\text {tot }}$, calculate $\vec{v}^{\text {old }}(k, M)$ and $\vec{v}^{\text {new }}(k, M)$, and obtain $\Delta \mu(k)$. Finally, $\Delta S_{\text {eff }}$ is evaluated from Eq. (40).

This procedure also gives error-free results if $\epsilon_{\mathrm{tr}}=0$.

Let us note that, the thresholds $\epsilon_{\mathrm{p}}$ and $\epsilon_{\mathrm{tr}}$ may be chosen independently, and the choice of these thresholds has to be justified by making proper estimates for the truncation errors.

Although there exists an arbitrary choice for the basis set to express the system in a quadratic form by Hamiltonian matrices (1), one should select that which reduces the propagation of the state vector as much as possible in order to reduce the computational complexities. A possible example is the case where there exists a symmetry in a system so that the Hamiltonian matrices may be block-diagonalized. The symmetry can be weakly broken as long as matrix elements between blocks are small. Then, using the basis set which block-diagonalize the Hamiltonian matrices, the vector propagations will be confined within the blocks.

Similarly, one may also make an extention to the algorithm so that the procedure for matrix products starts from an initial unit vector $v_{i}(k, 0) \neq \delta_{i k}$, provided the number of the non-zero vector elements in $\vec{v}(k, 0)$ is $O\left(N^{0}\right)$. An appropriate choice of the initial vector, typically an expression of local symmetries of the system, may cancel the propagation to some extent through interferences.

\subsection{Benchmark}

In order to demonstrate that the algorithm is successfully implemented, we show benchmark results. As a model, we choose the double-exchange (DE) model in the strong-coupling limit in three dimensions. The Hamiltonian is given by

$$
\hat{\mathcal{H}}\left(\left\{\vec{S}_{i}\right\}\right)=-\sum_{<i j>} t\left(\vec{S}_{i}, \vec{S}_{j}\right)\left(c_{i}^{\dagger} c_{j}+\text { h.c. }\right),
$$

where hoppings of spinless electrons to nearest neighbors are coupled to classical spin fields $\left\{\vec{S}_{i}\right\}$ in a form

$$
\frac{t\left(\vec{S}_{i}, \vec{S}_{j}\right)}{t_{0}}=\cos \frac{\theta_{i}}{2} \cos \frac{\theta_{j}}{2}+\sin \frac{\theta_{i}}{2} \sin \frac{\theta_{j}}{2} e^{-\mathrm{i}\left(\phi_{i}-\phi_{j}\right)} .
$$

Here $\theta$ and $\phi$ are defined by the direction of the localized spin $\vec{S}$ as

$$
S_{i}^{x}=S \sin \theta_{i} \cos \phi_{i}, S_{i}^{y}=S \sin \theta_{i} \sin \phi_{i}, S_{i}^{z}=S \cos \theta_{i},
$$

with the normalization $S=1$, while $t_{0}$ is the transfer integral between nearest neighbors in the absence of the DE interaction. A local update for $\vec{S}_{i}$ modulates all the hopping energies from $i$-th site to its nearest neighbor sites. Then, $\mathcal{C}$ for site $i$ contains nearest neighbors of $i$ as well as $i$ itself.

For parameters of the MC benchmark run, we choose $T / W \sim 0.02$ and $\mu / W \sim 0$ where $T$ and $\mu$ are temperature and the chemical potential, respectively, while $W=6 t_{0}$ is the half-bandwidth of the model in the absence of the interactions. We typically take MC steps as $N_{\text {step }} \sim 4000$. These parameters are typical ones for an investigation of the ferromagnetic transition in the model. ${ }^{3)}$ Within this parameter range, $M=16$ has been shown to be enough for the accuracy of the calculation.

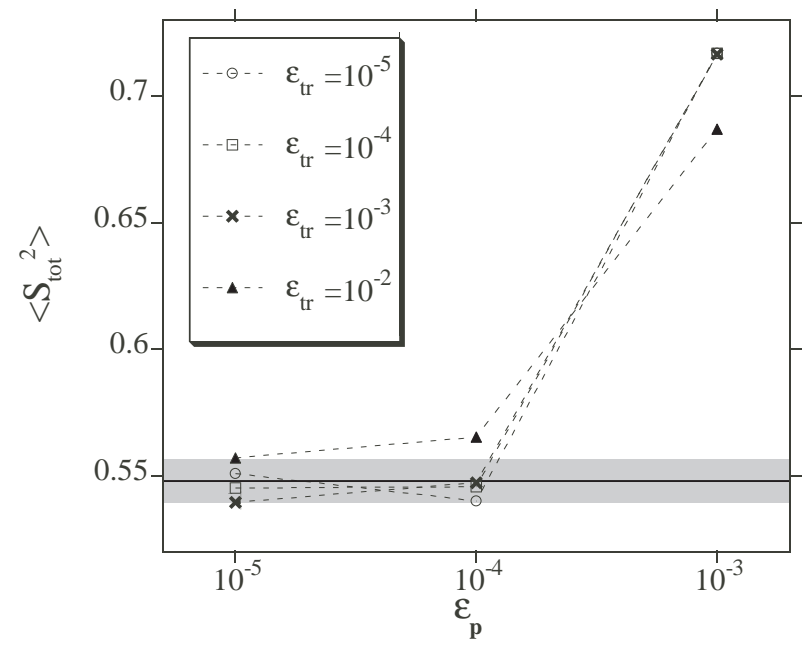

Fig. 3. Deviations of the estimated values due to truncations, for the square of the ferromagnetic moment $S_{\text {tot }}{ }^{2}$ on a $4 \times 4 \times 6$ lattice system at $T / W=0.015, \mu / W=0, M=16$ and $N_{\text {step }}=4000$. Symbols show estimates by the truncated PEM for various values of the truncation thresholds $\epsilon_{\mathrm{p}}$ and $\epsilon_{\mathrm{tr}}$. The solid line in the figure shows the estimated value of $S_{\text {tot }}{ }^{2}$ obtained by the DM, while the gray area around the line gives the stochastic error bar for the estimate. Error bars of each symbols are roughly equal to the error bar of the data by the DM. Therefore, if symbols are in the gray area, it is conceivable that overall truncation errors are roughly equal to or smaller than stochastic errors.

In Fig. 3, we show truncation errors for various combinations of $\epsilon_{\operatorname{tr}}$ and $\epsilon_{\mathrm{p}}$. We see that the truncation errors quickly decreases as the thresholds are lowered, and becomes sufficiently small compared to the statistical errors. From this result, we choose $\epsilon_{\mathrm{tr}}=10^{-3}$ and $\epsilon_{\mathrm{p}}=10^{-5}$ which are satisfactory to give accurate results with reduced cpu time in the present case. 
In usual cases, it is expected that we may take $\epsilon_{\mathrm{tr}}$ larger than $\epsilon_{\mathrm{p}}$. The threshold $\epsilon_{\mathrm{tr}}$ determines the border of the region $\mathcal{V}_{\epsilon_{\mathrm{tr}}}^{\text {tot }}$. For the indices at the border, contributions to $\Delta \mu_{m}(k)$ come from propagations from these indices to those in $\mathcal{C}$, which take small fractions of the whole propagations. On the other hand, the threshold $\epsilon_{\mathrm{p}}$ determines the border of all the propagations, including those from the indices near $\mathcal{C}$ which give relatively large values of $\Delta \mu_{m}(k)$. Therefore, $\Delta S_{\text {eff }}$ is more sensitive to the threshold $\epsilon_{\mathrm{p}}$.

Figure 4 shows the cpu time per MC step as a function of the system size on a single processor system as well as on a parallel computational system with the number of processor elements $N_{\mathrm{PE}} \leq 24$. From the figure we confirm that the cpu time is proportional to the system size. We also see that it is possible to implement this algorithm for efficient parallel computations.

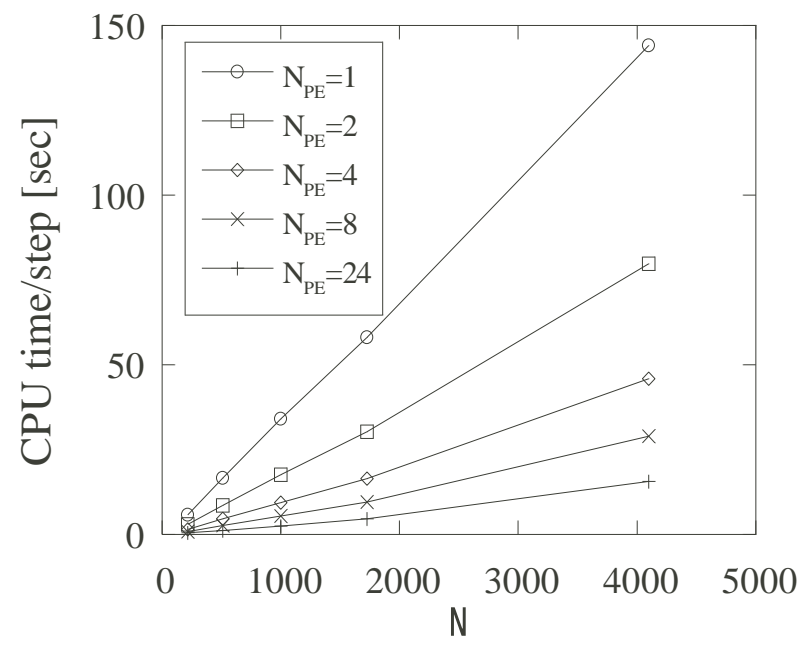

Fig. 4. Benchmark results for $6 \times 6 \times 6,8 \times 8 \times 8,10 \times 10 \times 10$, $12 \times 12 \times 12$ and $16 \times 16 \times 16$ sites of the double-exchange model in three dimensions, at $T / W=0.02, \mu / W=0$ and $M=16$, while $\epsilon_{\mathrm{tr}}=10^{-3}$ and $\epsilon_{\mathrm{p}}=10^{-5}$ are chosen for the thresholds. Computations are performed up to $N_{\mathrm{PE}}=24$ processor elements of an Athron MP1500+ cluster system which are connected by Myrinet 2000.

Table II gives benchmark results of the truncated PEM for various system sizes, in comparison with the diagonalization method and the full PEM. Using the truncated PEM, it becomes possible to calculate large size systems in a realistic time scale. For example, DE model in three dimensions up to $20 \times 20 \times 20$ becomes available, which is large enough to perform a finite-size scaling analysis of the critical phenomena in this model. The result is reported elsewhere. ${ }^{10)}$

\section{$\S 5$. Summary and Discussions}

Under the condition that the full PEM reduces the computational complexity for one $\mathrm{MC}$ step with local updates to $O\left(N^{3}\right)$ from that for the DM which is $O\left(N^{4}\right)$, namely, if we may take $M=O\left(N^{0}\right)$ to obtain accurate results, we have shown that the truncated PEM further reduces it to $O(N)$. It is possible to obtain the exact
Table II. An estimated cpu time of 10,000 MC steps for various system sizes of the DE model in three dimensions, at $T / W=$ 0.02 and $\mu / W=0$. For the PEM we have $M=16$, while $\epsilon_{\mathrm{tr}}=$ $10^{-3}$ and $\epsilon_{\mathrm{p}}=10^{-5}$ are chosen for the truncation thresholds. The cpu time is estimated on a personal computer with a single processor of the Athron MP1500+ processor.

\begin{tabular}{cccc}
\hline System size & Diagonalization & Full PEM & Truncated PEM \\
\hline $8 \times 8 \times 8$ & 2.3 years & 82 days & 2.4 days \\
$12 \times 12 \times 12$ & 300 years & 8.7 years & 8 days \\
$16 \times 16 \times 16$ & 9500 years & 120 years & 21 days \\
\hline
\end{tabular}

results within the PEM scheme if we take $\epsilon_{\mathrm{p}}=\epsilon_{\mathrm{tr}}=0$. The computational complexity can further be reduced by introducing non-zero values for $\epsilon_{\mathrm{p}}$ and $\epsilon_{\mathrm{tr}}$. The truncation errors can be made arbitrary small by taking small enough values for $\epsilon_{\mathrm{p}}$ and $\epsilon_{\mathrm{tr}}$.

So far we have restricted ourselves to systems with sparse Hamiltonian matrices as well as short-range interactions between adiabatical fields and electrons. When interactions are long ranged while the Hamiltonian matrices are still sparse, the PEM is applied with less efficiencies. A local update of the adiabatical fields modulates large numbers of Hamiltonian matrices, and therefore the number of indices in $\mathcal{V}_{\epsilon}^{\text {tot }}$ may be proportional to $N_{\text {dim }}$ in the procedure of the truncation of the trace operation. The truncation of the matrix-vector multiplication works similarly as before. Then, the computational complexity for a local update of the fields scales as $O\left(M^{\frac{d}{2}+1} N\right)$ instead of $O\left(M^{d+1}\right)$. Similar results will be obtained if one performs a global update of the adiabatical fields where number of the fields to be updated is $O(N)$.

However, in the case of systems with dense matrices, the PEM is completely inefficient. Namely, a multiplication of the Hamiltonian matrix make the vector to propagate to the whole space, so that procedures to restrict the subspace for calculations do not reduce computational complexities. Moreover, the matrix-vector multiplications cost $O\left(N_{\mathrm{dim}}^{2}\right)$ instead of $O\left(N_{\mathrm{dim}}\right)$. The full $\mathrm{PEM}$ as well as the truncated PEM gives $O\left(M N^{4}\right)$ computational complexities, and in this case, the DM should simply be used for calculations.

If we consider a case where calculations are performed on small size lattices, the propagation of the vectors quickly spreads to the whole space. In other words, the Hamiltonian matrices effectively become dense. In this case, the truncated PEM does not reduce the computational complexities in practice. In general, for each system there exists a minimum number for system sizes where the truncated PEM is avdantageous compared to the DM. The minimum number depends on the range of the vector propagations with $M$-steps, determined by model parameters as well as lattice dimensions and geometries.

Near the critical points, improved MC sampling techniques such as the histogram method or the multicanonical method are used to overcome the limitations of the importance sampling MC method. ${ }^{11)}$ These techniques 
can also be applied to the truncated PEM, where the energy of a sample is given by the "effective electronic free energy" defined by

$$
F_{\text {eff }}(\phi)=S_{\text {eff }}(\phi) / \beta
$$

Let us finally comment on the fact that $M$ may be kept to a constant which means that the propagations of electrons which contribute to $S_{\text {eff }}$ are limited to a finite distance $R_{0}(M)$. The situation is valid even at a critical point where correlation length for the classical fields diverges, since $S_{\text {eff }}$ are determined not by correlations but by the effective interaction energies among classical fields which may be short ranged. From the other point of view, actions are in general non-singular at critical points so that the polynomial expansion converges stably.

\section{Acknowledgment}

Calculations are performed on a AOYAMA+ system (http://www.phys.aoyama.ac.jp/ aoyama+/), parallel Athron MP1500+ PC computers connected by Myrinet 2000. The authors thank H. Nakata and M. Tsutsui for helpful supports in developing parallelcomputational systems. This work is supported by a Grant-in-Aid from the Ministry of Education, Culture, Sports, Science and Technology.

1) S. Yunoki, J. Hu, A. Malvezzi, A. Moreo, N. Furukawa and E. Dagotto: Phys. Rev. Lett. 80 (1998) 845.

2) Y. Motome and N. Furukawa: J. Phys. Soc. Jpn. 68 (1999) 3853.

3) Y. Motome and N. Furukawa: J. Phys. Soc. Jpn. 69 (2000) 3785; ibid 70 (2001) 3186.

4) J. Alonso, L. Fernandez, F. Guinea, V. Laliena and V. MartinMayor: Nuclear Phys. B596 (2001) 587.

5) Y. Motome and N. Furukawa: J. Phys. Soc. Jpn. 70 (2001) 1487.

6) Y. Motome and N. Furukawa: J. Phys. Soc. Jpn. 70 (2001) 2802.

7) L. Wang: Phys. Rev. B49 (1994) 10154.

8) R. Silver and H. Röder: Int. J. Mod. Phys. C5 (1994) 735.

9) N. Furukawa, Y. Motome and H. Nakata: Computer Phys. Comm. 142 (2001) 410.

10) Y. Motome and N. Furukawa: J. Phys. Soc. Jpn 72 (2003) 2126.

11) D. Landau and K. Binder: A Guide to Monte Carlo Simulations in Statistical Physics (Cambridge University Press, Cambridge, 2000). 